\title{
Effect of additives on lubrication properties of palm oil-based trimethylolpropane ester for hydraulic fluid application
}

\begin{abstract}
Hydraulic fluids are liquids used as the motive medium in hydraulic machinery and equipment. The current interest in protecting the environment has created a demand for vegetable-based and biodegradable hydraulic fluids. However, the lubricating properties of the vegetable-based such as poor oxidative stability and high pour point have hindered their use. In this study, Trimethylolpropane (TMP) Ester or TMPE, which is derived from palm oil-based methyl ester (POME), was used as the base fluid. The objective of this research is to study the lubrication properties of the formulated TMPE (TMPE $+1.0 \%$ of Additive A), namely the pour point (PP) test, wear and friction test and filterability test. The pour point obtained from this research was $10^{\circ} \mathrm{C}$ before applied to the lab-scale hydraulic test rig. This reading is much higher if compared to the commercial hydraulic fluid, which has the pour point of $-30^{\circ} \mathrm{C}$. Nevertheless, this result was slightly comparable to the unformulated TMPE, $12^{\circ} \mathrm{C}$. The wear test shows that at $15 \mathrm{~kg}$ load, the WSD was $1.0 \mathrm{~mm}$ before application (at 0 hour), with the coefficient of friction $(\mathrm{CoF})$ was 0.04 as compared to the WSD of unformulated oil, $0.46 \mathrm{~mm}$ and $\mathrm{CoF}$ of 0.04 . There is no data available at $15 \mathrm{~kg}$ load for the commercial hydraulic oil compared. Meanwhile, at $40 \mathrm{~kg}$ load, the WSD recorded was 3.24 $\mathrm{mm}$ before the application compared to commercial hydraulic oil, $0.36 \mathrm{~mm}$ and unformulated oil, $0.64 \mathrm{~mm}$. After 800 hours application, the WSD increase sharply both for $15 \mathrm{~kg}$ (1049 $\mathrm{mm})$ and $40 \mathrm{~kg}(3364 \mathrm{~mm})$. It showed that there were particles built in the formulated oil. This is supported by the filterability study.
\end{abstract}

Keyword: Filterability; Hydraulic fluid; Lubrication; Palm oil; Pour point; Wear 